\title{
Functional connectivity of the amygdala is linked to individual differences in emotional pain facilitation
}

Article

Accepted Version

Gandhi, W., Rosenek, N. R., Harrison, R. and Salomons, T. V. (2020) Functional connectivity of the amygdala is linked to individual differences in emotional pain facilitation. PAIN, 161 (2). pp. 300-307. ISSN 0304-3959 doi:

https://doi.org/10.1097/j.pain.0000000000001714 Available at https://centaur.reading.ac.uk/88776/

It is advisable to refer to the publisher's version if you intend to cite from the work. See Guidance on citing.

Published version at: http://dx.doi.org/10.1097/j.pain.0000000000001714

To link to this article DOI: http://dx.doi.org/10.1097/j.pain. 0000000000001714

Publisher: Pain

All outputs in CentAUR are protected by Intellectual Property Rights law, including copyright law. Copyright and IPR is retained by the creators or other copyright holders. Terms and conditions for use of this material are defined in the End User Agreement.

www.reading.ac.uk/centaur 
Central Archive at the University of Reading

Reading's research outputs online 
Functional connectivity of the amygdala is linked to individual differences in emotional pain facilitation

Wiebke Gandhi ${ }^{1}$, Norma R. Rosenek ${ }^{1}$, Richard Harrison ${ }^{1}$, Tim V. Salomons ${ }^{1,2}$

1) School of Psychology and Clinical Language Sciences, University of Reading, Reading UK

2) Department of Psychology, Queen's University, Kingston (Ontario), Canada

Number of pages (including figures and tables): 39

Number of figures: 4 (displayed at the end of this document)

Number of tables: 2 (displayed at the end of this document)

\section{Corresponding authors:}

Tim Salomons, Ph.D.

Department of Psychology

Queen's University

62 Arch Street, Kingston, ON

K7L 3N6

Canada

E-mail address:

tim.salomons@queensu.ca 


\section{Abstract}

The amygdala is central to emotional processing of sensory stimuli, including pain. Because recent findings suggest that individual differences in emotional processes play a part in the development of chronic pain, a better understanding of the individual patterns of functional connectivity that make individuals susceptible to emotionally modulated facilitation of pain is needed. We therefore investigated the neural correlates of individual differences in emotional pain facilitation using resting-state functional magnetic resonance imaging (rsfMRI) with amygdala seed.

Thirty-seven participants took part in 3 separate sessions, during which pain sensitivity was tested (session 1), participants underwent rs-fMRI (session 2), and emotional pain modulation was assessed (session 3). Amygdala served as seed for the rs-fMRI analysis and whole-brain voxelwise connectivity was tested. Pain modulatory scores were entered as regressor for the group analysis.

Stronger connectivity of the amygdala to S1/M1, S2/operculum, and posterior parietal cortex at rest characterized individuals who showed greater pain facilitation by negative emotions. When comparing the amygdala networks associated with pain unpleasantness and with pain intensity modulation, most of the identified areas were equally related to either pain rating type; only amygdala connectivity to S1/M1 was found to predict pain intensity modulation specifically.

We demonstrate that trait-like patterns of functional connectivity between amygdala and cortical regions involved in sensory and motor responses are associated with the individual amplitude of pain facilitation by negative emotional states. Our results are an early step towards improved understanding of the mechanisms that give rise to individual differences in emotional pain modulation. 


\section{Introduction}

Pain is a complex experience that is perceived differently across people and varies even within the individual depending on context [9]. Emotional states, for instance, modulate pain experiences, with positive mood attenuating [31; 46; 62; 79; 80;83], and aversive events facilitating it $[6 ; 34 ; 57]$. The close relationship between emotions and pain is reflected in the high rate of comorbidity between pain syndromes and affective disorders [4; 44]. Depressed individuals are twice as likely to develop chronic pain compared to nondepressed people $[11 ; 51]$. Conversely, chronic pain is frequently accompanied by anhedonia-like symptoms, including fatigue and reduced involvement in formerly pleasurable activities [66].

From previous findings we know that the amygdala plays a key role in the emotional evaluation of sensory stimuli $[16 ; 18 ; 50]$, including pain $[20 ; 48]$, and even seems to tag the incoming nociceptive signal as aversive before it is being further processed by cortical brain areas [14]. Moreover, activation of the amygdala has been shown to be correlated with the modulation of pain perception [58] and behavioural responses to pain [64] following the presentation of positively and negatively valenced stimuli. Notably, amygdala activity during regulation of aversive but non-painful affective states predicts individual differences in the ability to regulate pain [38]; therefore, the amygdala seems a critical link between pain and emotion regulation. Moreover, brain structures implicated in emotion-regulation, including amygdala, have recently been associated with the chronification of pain [25]. Hashmi and colleagues showed that, compared to people with 'subacute' pain (i.e. pain experienced for less than three months), chronic pain patients (with persistent pain for six or more months) had more pain-related activation in the amygdala and medial prefrontal cortex. Additionally, 
the authors documented a longitudinal shift in pain-related activation from nociceptive circuitries to emotion-related circuitries - including amygdala - as subacute pain evolved into chronic pain [25]. Because these studies suggest that individual differences in emotional processes may play a part in the development of chronic pain, a better understanding of trait-like patterns of neural connectivity that make some individuals susceptible to emotionally modulated facilitation of pain is needed. In the current study we therefore investigated the neural correlates of individual differences in emotional facilitation of pain using resting-state functional magnetic resonance imaging ( $r s-f M R I)$ with a bilateral amygdala seed. We hypothesised greater modulation of pain by negative emotion would be associated with greater connectivity of the amygdala to a network comprised of cortical regions that are functionally connected to the amygdala [61] and that are typically involved in processing the salience of sensory input, specifically the ACC/aMCC, operculum/insula, and parietal cortex $[9 ; 15 ; 30 ; 54]$. 


\section{Methods}

\section{Participants}

Of 40 initially included participants, three were excluded from the final analysis due to incomplete questionnaire data $(n=1)$, and excessive head movement during the scanning procedure (more than $2.5 \mathrm{~mm}, \mathrm{n}=2$ ). Consequently, our final study sample consisted of 37 young healthy participants ( 15 women; $23 \pm 5$ years in age). All participants had no history or presence of any chronic pain disorder, substance abuse, major medical or psychiatric disorder; none of them met any of the MRI exclusion criteria and all had normal or correctedto-normal vision. The study was approved by the local Ethics Committee at the University of Reading (UREC) and written consent was obtained from all participants according to the revised Declaration of Helsinki (2013).

\section{Experimental Procedures}

The data presented in the current manuscript was collected as part of a larger study, which included four experimental sessions.

Prior to the first session, participants completed three questionnaires, including the Pain Catastrophizing Scale (PCS)[72], Beck's Depression Inventory (BDI-II) [5], and the state anxiety scale of the State-Trait Anxiety Inventory (STAI) [69], on a secure 3rd-party website (https://www.surveymonkey.com). In the first experimental session we performed quantitative sensory testing in all participants (not reported in the current manuscript) and assessed their thermal pain threshold. The second session took place within seven days of the first session, and participants underwent brain imaging, including the rs-fMRI scan, as well as 
an anatomical scan (for the purposes of registration) and an event-related EPI scan, which was completed after the rs-fMRI scan. Finally, during session three and four we examined participants' pain modulation using emotional and cognitive pain-modulatory tasks. Sessions 3 and 4 were completed within a maximum of 2 weeks from the initial assessment - the entire study (sessions 1-4) were all completed within a maximum of 3 weeks. For the current analysis we will report the emotional pain-modulatory task (see below) exclusively. Our group has previously published a paper on neural correlates of trait mindfulness from this same data set [23].

\section{Estimation of pain thresholds (session 1)}

Using a MEDOC Pathway system with a 30x30mm Peltier thermode (Medoc Medical Systems, Ramat Yishai, Israel), we applied thermal stimuli to the participant's right leg; the stimulated leg was placed comfortably in an in-house designed leg rest. Individual pain thresholds were assessed using two approaches, i.e. method of limits and method of levels. For the method of limits the temperature increased from a baseline temperature of $32^{\circ} \mathrm{C}$ at a rate of $0.5^{\circ} \mathrm{C}$ per second, until the participant indicated that the stimulus had just become painful. Each participant performed 4 repetitions in total, with $15 \mathrm{~s}$ in between each stimulus. The method of limits threshold was defined by the average temperature indicated over the final 3 trials. Similarly, for the method of levels the baseline temperature was set at $32^{\circ} \mathrm{C}$. It was then increased by $8^{\circ} \mathrm{C}$ (at a rate of $2^{\circ} \mathrm{C} / \mathrm{s}$ ), before the temperature returned back to baseline (at a rate of $\left.-2^{\circ} \mathrm{C} / \mathrm{s}\right)$. After the application of the stimulus, participants indicated whether they had perceived it as painful. If they indicated no pain, the following stimulus was increased in temperature by an additional $2^{\circ} \mathrm{C}$. Conversely, if they did indicate pain, the temperature of 
the following stimulus was decreased at half the interval size; this procedure was applied until four reversals in direction had been reached. The method of levels threshold was defined by the average temperature across the last two trials. Finally, we determined the participant's mean pain threshold by averaging the temperatures of the limits and levels threshold.

To obtain the temperature to be applied within the emotional pain-modulatory task, participants were exposed to a series of thermal stimuli of eight different intensities around their individual pain threshold (PT), three below PT, PT, and four above PT - specifically, PT minus $2.25^{\circ} \mathrm{C}$; PT minus $1.50^{\circ} \mathrm{C}$; PT minus $0.75^{\circ} \mathrm{C}$; PT; PT plus $0.75^{\circ} \mathrm{C}$; PT plus $1.50^{\circ} \mathrm{C}$; PT plus $2.25^{\circ} \mathrm{C}$; PT plus $3.00^{\circ} \mathrm{C}$. Each temperature was repeated three times (with a stimulus duration of $8 \mathrm{~s}$, and an inter-stimulus interval of $30 \mathrm{~s}$ ) and applied in pseudo-random order to the middle of the lower calf of the right leg - the identical stimulation site was used again in session 3 during the emotional pain modulation task (see below). Although applied temperatures naturally differed across participants (dependent on their PT), the order of the eight different stimulus intensities remained constant across participants. Temperatures for the mid-point of the series (i.e. the temperature corresponding to PT) were rounded up or down to the next half number, e.g. if the pain threshold was estimated to be at $43.3^{\circ} \mathrm{C}$ the corresponding mid-point would have been $43.5^{\circ} \mathrm{C}$; accordingly, for a pain threshold of $43.2^{\circ} \mathrm{C}$ the corresponding mid-point would have been $43.0^{\circ} \mathrm{C}$. Across participants, we used 8 different mid-points, with $42^{\circ} \mathrm{C}$ being the lowest, and $45.5^{\circ} \mathrm{C}$ the highest. After each stimulus, participants rated the pain intensity they had perceived on a numerical rating scale with 0 indicating "no pain" and 10 representing "most intense pain imaginable" [23; 86]. Although participants were asked for verbal ratings, there were physical versions of the numerical rating scale on the wall they faced - this scale was identical to the one they would be presented with on computer screen in session 3 (see below). Based on these ratings, we 
estimated an individual stimulus response curve using an online regression calculator (http://www.alcula.com/calculators/ statistics/linear-regression/) that interpolated the temperature corresponding to a pain intensity rating of 6 out of 10 . This individually adjusted temperature was then used for the pain stimulation within the emotional painmodulation task. Before commencing the emotional pain modulation task (session 3, see below), we applied a test stimulus to the participants' right leg, using the temperature identified in session 1. If participants rated this test stimulus between 5 and 7 out of 10, we continued with the emotional pain-modulation task. However, if participants rated the stimulus below or above this range, we adjusted the temperature accordingly in steps of $\pm 0.5 \circ \mathrm{C}$ and repeated the test stimulus until a moderate pain rating was reached (i.e. 5 7/10).

\section{MRI data acquisition (session 2)}

Within the scanning session, we completed a rs-fMRI scan, a structural scan, and an eventrelated $\mathrm{fMRI}$ scan, including noxious stimuli. The noxious stimuli were administered after the completion of the resting state, with the aim of collecting rs-fMRI data, without the potential influence of noxious stimuli impacting the participant. No emotional images were used during session 2. For the purpose of this study, we will hereafter focus on the rs-fMRI and the structural scan exclusively - one article on this data set has already been published [23] and the remaining data will be published elsewhere.

Brain images were acquired using a 3 T Siemens Magnetom TRIO scanner (Siemens, Erlangen, Germany) with a standard 32-channel head coil. 
Participants were asked to close their eyes and rest, while functional MRI data were acquired using a blood oxygenation level-dependent (BOLD) protocol with a $\mathrm{T} 2 *$-weighted gradient echo planar imaging (EPI) sequence $\left(T E=28 \mathrm{~ms}, T R=2000 \mathrm{~ms}\right.$, flip angle $=90^{\circ}, 1 \mathrm{~mm}$ interslice gap; $128 \times 128$ matrix, field-of-view $=240 \mathrm{~mm}$ ). Per volume, thirty interleaved sagittal slices (3.5mm each) were acquired, covering the entire brain. To allow for steady-state magnetization the first three volumes were discarded. Subsequently, 300 volumes were acquired, equalling a scan time of 10 minutes in total. Anatomical images were acquired using a 6-minute T1-weighted inversion recovery fast gradient echo-high resolution structural scan (176 volumes, $\mathrm{TE}=2.9 \mathrm{~ms}, \mathrm{TR}=2000 \mathrm{~ms}, \mathrm{FA}=90^{\circ}$, voxel size $=1 \times 1 \times 1 ; 256 \times 256$ matrix, $\mathrm{FOV}=250 \mathrm{~mm})$.

\section{Assessment of emotional pain-modulation (session 3)}

Participants sat in a chair resting their right leg in a leg-rest with the thermode touching their mid-calf.

To assess emotional pain modulation, we used a behavioural paradigm similar to previously published ones [3; 31; 33]. Specifically, in each trial of the emotional pain-modulation task (fig. 1), participants were presented with five images drawn from the International Affective Picture System (IAPS) database [35]. On each trial, the five images were either of negative valence (in 18 trials) or of neutral content (also in 18 trials), alternating across trials. These images were drawn from a pool of 45 negative images and 45 neutral images; thus, each image was presented twice across the experiment. After 18 trials, participants had a short break, before commencing the second block of the remaining 18 trials. Within each trial, each of the five images was presented for $6 \mathrm{~s}$, with no delay in between the different 
images. With the presentation of the fourth and fifth image, the pain stimulus was simultaneously applied for the duration of $12 \mathrm{~s}$. At the end of each trial, participants were asked to rate pain intensity and unpleasantness on numeric rating scales ranging from 0 to 10, with 0 indicated "no pain" /"not at all unpleasant" and 10 "most intense pain imaginable"/"extremely unpleasant". The scales were presented subsequently on the computer screen within the E-Prime program and participants used the arrow keys to move an indicator on the screen in 0.5 steps to the respective numbers ( 0 through 10$)$. The completion of the task took approximately 30 minutes.

The IAPS images for each condition of the pain modulation task were chosen based on valence and arousal ratings of a norm population [37], using the Self-Assessment Manakin (SAM) tool by Lang (1980) [36] - a manakin tool, that was encoded into a 9-point scale with 1 being very negative in valence, 5 neutral, and 9 very positive. For the arousal scale, higher arousal was encoded as higher numbers (i.e. 1 represents no arousal and 9 high arousal). Based on the norm population, the mean valence rating for the neutral condition in our task was $4.85(S D=1.11)$, and mean arousal $2.61(S D=1.86)$, while the mean valence for the negative condition was $2.14(\mathrm{SD}=1.43)$, and mean arousal $6.38(\mathrm{SD}=2.19)$.

To assess rating behaviour in our sample, we asked participants to rate valence and arousal in response to all negative images we used. This was done separately to the actual task (i.e. without simultaneous pain presentation), in three blocks - once before commencing the emotional pain modulation task, once during the break and once the task was finished. Each time, participants were presented with ten of the thirty negative images that were used in the task and rated each of them on the SAM tool. To assess that our participants showed expected rating behaviour, we compared their ratings to the ratings of the IPAS norm data 
set (Lang, Bradley, \& Cuthbert, 2005) for the respective images. To confirm that our sample and the norm population were indeed not statistically different, we z-transformed the raw values of the mean ratings across all 30 negative images for each participant $\mathrm{z}=\left(\right.$ mean $\left._{\mathrm{i}}-\operatorname{mean}_{\text {norm population }}\right) / \mathrm{SD}_{\text {norm population }}-\mathrm{using}$ the corresponding norm population's mean and standard deviation from the IAPS norm data set (Lang, Bradley, \& Cuthbert, 2005).

\section{Data Analysis}

\section{Behavioural Data Analysis}

To test the influence of valence condition of the visual stimuli on pain ratings we performed a $2 \times 2$ repeated measures ANOVA with valence condition ( 2 levels: neutral and negative) and pain rating type ( 2 levels: pain unpleasantness and intensity) as the two within-subject factors, and pain rating as the dependent variable. Significant interaction effects were followed by separate paired Student's t-tests between the valence conditions of the visual stimulus (neutral and negative) for each of the two rating types (pain unpleasantness and pain intensity).

The significance level was set to $p<0.05$ for all analyses. Across all analyses, outliers were defined as exceeding the group mean value by more than 2 standard deviations and were excluded from the analyses. This only occurred once. Before averaging the pain intensity ratings across negative trials, we identified the average across the sample for the first trial to be more than 2 SD lower than the remaining trials. Thus, trial 1 was excluded from the 
average of pain intensity ratings in both valence conditions. All statistical analyses were performed using IBM SPSS Statistics 24 (SPSS Inc. Chicago, USA).

\section{$\underline{\text { Resting state-fMRI data analysis }}$}

Seed Selection

Bilateral amygdala was selected as the seed for our seed-based whole brain connectivity analysis (fig. 2) due to its prominent role in the modulation of pain by emotion [58; 64]. Both amygdalae were anatomically defined based on the Harvard-Oxford Subcortical Structure Atlas implemented in FSL (using a probabilistic threshold of 25\%) and combined to one single mask of bilateral amygdala using the FSL tool 'Fs/maths'(www.fmrib.ox.ac.uk/fsl, [28]). Because laterality effects for amygdala function have been previously shown for pain processing in rodents [65] and humans [71] we first tested whether the left and right amygdalae were associated with different patterns of emotional pain modulation. However, upon visual inspection of our results, there were no differences in functional connectivity between left and right amygdala seeds for either type of emotional modulation of pain (intensity or unpleasantness). Therefore, only the significant activation clusters for the bilateral seed are reported in the results section of our article.

\section{Pre-Processing}

Pre-processing was performed using FSL 6.00 (www.fmrib.ox.ac.uk/fsl, [28]). For skull stripping we used the Brain Extraction Tool (BET) [68]. To allow for signal equilibration we discarded the first five volumes. For the remaining data, an interleaved slice-timing correction 
was applied, and data were spatially smoothed with a $5 \mathrm{~mm}$ full-width half-maximum (FWHM) Gaussian kernel. Data were motion corrected using MCFLIRT [27] and visually examined for further motion artifacts, problems with the registration or poor skull stripping.

Grey matter was segmented from white matter (WM) and cerebrospinal fluid (CSF) using FAST [88]. Thresholds of 0.99 were applied for WM and CSF maps. Time series were extracted for WM and CSF and entered into a general linear model in addition to motion parameters. Residuals from this nuisance analysis were normalised and bandpass filtered at $0.1 / 0.01 \mathrm{~Hz}$ to diminish the influence of low frequency drifts (including scanner drift), as well as high frequency artifacts such as cardiac or respiratory interferences.

Resting State fMRI Analysis

The mean time series of all voxels within our amygdala seed region were extracted and included as a regressor in a whole-brain functional connectivity analysis. Contrast images were then entered to higher level analyses in which participants' demeaned pain modulatory scores were added as a regressor. Because we were interested in the individual variance in pain unpleasantness and intensity modulation across the group (fig. S1) and its association to amygdala connectivity at rest, pain modulation scores were calculated separately for pain intensity and unpleasantness, where each was the difference between mean ratings in the negative and neutral valence conditions. The aim of this analysis was to identify regions where connectivity with bilateral amygdala was significantly associated with pain modulation by negatively-valenced stimuli. 
Analyses were corrected for multiple comparisons using Gaussian random field theory $(Z<2.3 ; p<0.05)$. Parameter estimates were extracted from regions where functional connectivity with the amygdala was significantly correlated to pain modulation using FEATquery as implemented in FSL. Parameter estimates were extracted for the significant clusters as identified by the statistical parametric maps of our GLM analysis. To restrict the identified clusters to grey matter of the respective brain regions that were associated with emotional pain modulation, we multiplied them with anatomical masks of the grey matter of those regions as defined by the 'Harvard-Oxford Cortical Structure Atlas' implemented in FSL (using a probabilistic threshold of $25 \%$ for each anatomical mask). Extracted parameter estimates for each of these regions were used to depict the correlation between them and our behavioural measures of pain modulation (pain intensity and unpleasantness) by emotion. Similar to the behavioural data, parameter estimates were also correlated to the questionnaire data using Pearson's product-moment correlation coefficients.

Finally, to test the equality of these correlations between the two types of pain ratings (i.e. modulation of pain unpleasantness versus intensity) under consideration of the correlation between pain unpleasantness and pain intensity ratings, we used a modification of Hotteling's $T_{1}$ [85] that has previously been recommended for the comparison of two correlations for sample sizes comparable to ours [70]. The applied formula was

$T 2=\left(r_{x z}-r_{y z}\right) V\left(\left((N-1)\left(N+r_{x y}\right)\right) /\left(2((N-1) /(N-3))|R|+\check{r} 2\left(1-r_{x y}\right) 3\right)\right)$

where $\check{r}=1 / 2\left(r_{x z}+r_{y z}\right)$, and $|R|=\left(1-r_{x z} 2-r_{y z} 2-r_{x y} 2\right)+\left(2 r_{x z} r_{y z} r_{x y}\right) ;$ $r_{x y}$ representing the correlation coefficient between the two pain rating types (pain unpleasantness and pain intensity), 
$r_{x z}, r_{y z}$, the correlation coefficient between the connectivity findings and each of the pain rating types, and $\mathrm{N}$ being the number of participants.

T2 has a t-distribution with $\mathrm{N}-3$ degrees of freedom [70]. 


\section{Results}

Quality control: participants showed expected rating behaviour for negative IAPS pictures.

Participants in our study rated the valence of the negative IAPS pictures on average as 2.81 $(S D=0.96)$, which was comparable to the norm population's rating (mean=2.14, $S D=1.43$, $z=0.47, p=0.64)$. They further rated arousal on average as $6.22(S D=1.35)$, which was also comparable to the norm population's rating (mean $=6.38, S D=2.19, z=-0.07, p=0.94$ ). The analysis thus confirmed that participants in this study rated the IAPS pictures as expected, with no difference to the norm population.

Visual stimuli of negative valence modulate pain perception.

Pain intensity and pain unpleasantness ratings were positively correlated in both conditions, with $r=0.89(p<0.001)$ in the neutral condition, and $r=0.88(p>0.001)$ in the negative condition.

Pain ratings were significantly influenced by the valence of the simultaneously presented images and pain rating type in an interacting fashion (interaction effect of 'valence by rating type' $\left.F_{1,36}=8.30, p<0.01\right)$. The post-hoc paired t-tests further revealed that pain unpleasantness ratings were significantly higher in the negative valence condition than in the neutral one $-M_{\text {neutral }}=5.08(S D=1.96), M_{\text {negative }}=5.70(S D=1.81), t_{36}=2.82, p<0.01 . \ln$ contrast, pain intensity ratings were not significantly different between the valence conditions $-M_{\text {neutral }}=5.53(S D=1.81), M_{\text {negative }}=5.63(S D=1.70), t_{36}=1.02, p=0.32$. 
Functional connectivity of the amygdala underlying the magnitude to which individuals modulate pain by emotion

The rs-fMRI analysis showed that individuals with a greater increase in pain unpleasantness ratings when viewing negatively valenced images had stronger functional connectivity of the amygdala to a network encompassing bilateral posterior operculum/S2 and central operculum (extending to anterior insula in the right hemisphere), and the right inferior parietal cortex (fig. 2; table 1 - for detailed statistical maps of amygdalar connectivity correlated with emotional pain modulation see fig. S2). Repeating the analysis for functional connectivity of the amygdala underlying the individual magnitude to modulate pain intensity by valence revealed a network of brain areas including bilateral S1/M1, and bilateral posterior and central operculum/S2 (fig. 2; table 1). No correlations were found between the scores of the included questionnaires (PCS, BDI-II, and STAI) and our connectivity data (all $|r|<0.27$, all $p>0.06$; see table S1 for more detail).

Equality of the amygdala network for the modulation of pain unpleasantness vs intensity To test whether these associations were specific to the sensory or emotional component of pain modulation, we compared the correlations between amygdala connectivity and emotional pain modulation for the different pain rating types (unpleasantness versus intensity); this analysis revealed a significantly greater correlation for amygdala-S1/M1 connectivity with the emotional modulation of pain intensity ratings than with emotional modulation of pain unpleasantness $\left(r_{x z}=0.711, r_{y z}=0.504\right.$, respectively; $\left.T_{2}(32)=-2.97, p<0.01\right)$. Amygdala connectivity to all other identified regions - i.e. right inferior parietal cortex (hereafter referred to as 'parietal cortex' for simplicity), bilateral operculum/S2 (hereafter referred to as 'S2' for simplicity), and right central operculum - showed comparable 
correlations for both pain rating types. The results suggest that amygdala-S1 connectivity is a specific marker for modulation of sensory aspects of pain, while all other associations are non-specific.

Table 1 Brain regions associated with emotional modulation of pain intensity or pain unpleasantness

\begin{tabular}{|c|c|c|c|c|c|c|}
\hline \multirow[t]{2}{*}{ Seed } & \multirow{2}{*}{$\begin{array}{l}\text { Behaviour } \\
\text { al } \\
\text { Measure }\end{array}$} & \multirow[t]{2}{*}{ Brain region } & \multicolumn{3}{|c|}{ MNI coordinates } & \multirow{2}{*}{$\begin{array}{l}\text { Peak } \\
\text { z- } \\
\text { score }\end{array}$} \\
\hline & & & $x$ & Y & Z & \\
\hline & & Positive correlation & & & & \\
\hline \multirow[t]{3}{*}{$\begin{array}{l}\text { Bil. } \\
\text { Amygdala }\end{array}$} & Pain UP & $\begin{array}{l}\text { Parietal/central operculum } \\
\text { (R) }\end{array}$ & 36 & -4 & 18 & 4.00 \\
\hline & & Parietal operculum (L) & -32 & -40 & 24 & 3.74 \\
\hline & & Inferior parietal cortex (R) & 64 & -34 & 18 & 3.25 \\
\hline \multirow{5}{*}{$\begin{array}{l}\text { Bil. } \\
\text { Amygdala }\end{array}$} & Pain & Parietal operculum (R) & 54 & -28 & 28 & 4.09 \\
\hline & Intensity & & & & & \\
\hline & & Parietal operculum (L) & -56 & -32 & 20 & 3.81 \\
\hline & & $\mathrm{M} 1 / \mathrm{S} 1(\mathrm{R})$ & 48 & -8 & 44 & 4.08 \\
\hline & & $\mathrm{M} 1 / \mathrm{S} 1$ (L) & -54 & -8 & 42 & 3.80 \\
\hline
\end{tabular}




\section{Discussion}

This study examined whether resting state functional connectivity of the amygdala can predict the level to which an individual is prone to emotional facilitation of pain. We observed that a stronger connectivity of the amygdala to S1/M1, S2, central operculum, and posterior parietal cortex characterized individuals who showed greater facilitation of pain by negative emotions. Connectivity of most of the identified areas was equally related to pain unpleasantness and pain intensity; only amygdala connectivity to S1/M1 was found to predict pain intensity modulation to a significantly greater degree than pain unpleasantness modulation and, as such, might selectively code an individual's propensity for emotional modulation of the sensory dimension of pain.

Mood and emotions influence the pain experience, with negatively valenced framings leading to an exacerbation of pain $[78 ; 79 ; 84]$. Interestingly, a negative emotional state predominantly increases the perceived unpleasantness of a simultaneously applied painful stimulus without necessarily altering its intensity (for review [9]). In our study, we replicated the effect of pain unpleasantness exacerbation by elicited negative emotions.

Demonstration of a significant facilitation of pain unpleasantness by negative emotion could lead to the interpretation that this effect is observed uniformly across individuals. It should therefore be noted that we observed considerable variation in the degree of pain modulation by negatively valenced images, with some experiencing no modulation, or even reduction of pain. This spread indicates that there are substantial individual differences in vulnerability to emotional facilitation of pain.

In this study, we focussed specifically on the neural mechanisms that characterize individuals who are prone to emotional facilitation of pain. More specifically, we investigated individual differences in the connectivity of the amygdala, because of its role in 
the emotional evaluation of pain $[14 ; 20 ; 48]$, and pain chronification $[14 ; 25]$. Historically, it has been suggested that the intensity and location of nociceptive input is encoded in brain areas including S1, S2/operculum, and posterior insular cortex ([12; 13; 22; 52; 53; 76]. In contrast, the emotional aspect of pain has been associated with brain areas such as the anterior cingulate cortex, posterior parietal cortex and anterior insula (e.g. [53; 55; 75; 89], integrating neural input from sensory brain areas and limbic structures, including the amygdala [53; 76]. More recent studies show, however, that this strict distinction between the two divisions of sensory-discriminative and emotional pain processing is an oversimplification, with many of these brain regions being in fact sensitive to both aspects [82]. This study demonstrates how the interplay of these two sets of regions might determine how sensory and emotional information interact in an individual's experience of pain. Importantly, in this respect, most of the structures within the identified network were not uniquely linked to the emotional modulation of pain intensity or unpleasantness but showed comparable correlations to both pain rating types.

While resting state functional $\mathrm{fMRI}$ is useful for detecting trait-like patterns of connectivity associated with psychological phenomena [47; 52; 75], it gives limited information about how these patterns of connectivity are involved in those phenomena. Nevertheless, previous work can help us form preliminary hypotheses about the processes the connected regions are engaged in, and how these processes might influence behavioural and emotional responses. The amygdala's role in emotional priming for fear- and anxietytriggering stimuli is well known $[17 ; 40 ; 42 ; 43 ; 50]$. Given that the amygdala receives nociceptive input through the spino-parabrachio-amygdaloid pathway [74], it is likely that the amygdala plays a similar role in emotional priming for nociceptive input, to allow for a nocifensive reaction before the stimulus has even reached consciousness. In fact, a recent 
study in rodents provides evidence that activation of a neural ensemble specific to nociceptive processing within the basolateral nucleus of the amygdala predicts the magnitude of subsequent nocifensive behaviour [14]. The amygdala's role in nocifensive behaviour is further supported by a study in humans by Roy and colleagues [63]. The authors found that simultaneous presentation of pictures of negative versus neutral content not only increased pain ratings (modulation of pain unpleasantness by emotion was not assessed in this study), but also the amplitude of the nociceptive flexion reflex - a withdrawal reflex of the potentially endangered extremity. In contrast to their study, we did not find a significant effect of emotional stimulation on pain intensity ratings, but on pain unpleasantness. Given that the two rating types are highly correlated though, it can be assumed that the results in the referenced study are still of relevance to our results. Specifically, the findings by Roy and colleagues [63] imply an increased vigilance of the body towards painful stimuli when the organism is in a negative emotional state, resulting in a facilitation of preconscious pain actions. Importantly, the emotional modulation of the nociceptive flexion reflex covaried with bilateral amygdala activation [64], demonstrating the amygdala's role in preparing the individual for nocifensive responses on the basis of emotional state.

While this initial spinal reflex provides a rapid action to prevent harm, the amygdala's afferent input from cortical sources and efferent connectivity to descending modulatory regions like the PAG $[41 ; 49 ; 74]$ would also facilitate more complex, cortex-regulated behaviours involved in controlled and voluntary defence responses, such as approaching and tackling the source of pain or moving away from the harmful environment [47]. The posterior parietal cortex plays an important role in sensorimotor transformation of threatrelevant visual stimuli $[8 ; 19 ; 60]$ while primary sensory and motor cortex carry out the 
appropriate final motor action $[29 ; 56 ; 67]$. As such, in our study, connectivity of the amygdala with these regions may represent a preparedness for emotionally facilitated responses to nociceptive input, both conscious and pre-conscious.

The relationship between the connectivity within this affective-sensorimotor network and the described regulatory defence mechanisms might follow a Yerkes-Dodson curve, portraying an inverse $U$-shaped relation between arousal and adaptive response [87] - a relationship that relies on intact amygdala functioning as shown by rodent studies (reviewed in [1]). People with a generally increased connectivity within this affective-sensorimotor network might activate the described regulatory defence mechanisms faster when perceiving pain in an emotionally laden environment, resulting in efficient and adaptive pain-defence behaviours in individuals with moderately increased connectivity. However, individuals with hyper-connectivity within the described affective-sensorimotor network could well be at risk for maladaptive nocifensive responses, which in turn, might sensitise the individual to further pain. An example where the relationship between emotional state and adaptive/maladaptive pain behaviours might be particularly relevant is the transition from acute to chronic pain in the wake of physical trauma, such as surgery. In fact, depressed individuals - who are naturally shifted towards higher aversive emotional levels are twice as likely to develop chronic pain compared to non-depressed people $[11 ; 51]$; they have poorer outcomes in response to pain treatments, describe diminished life-quality, and require higher treatment costs [10; 32]. Furthermore, affective/emotional distress including depression $[7 ; 24 ; 26 ; 77 ; 81]$ and anxiety $[21 ; 26 ; 45 ; 73 ; 77 ; 81]$ - as well as maladaptive pain-related cognitions - such as pain catastrophizing $[26 ; 73 ; 81]$ - have been repeatedly shown to be predictors for the chronification of pain following surgery $[2 ; 39$; 59]. These results suggest an interacting effect of psychological factors and physical trauma 
(e.g. surgery) on the chronification of pain, with psychological/emotional states shaping the response to post-operative pain. An intriguing question that arises is whether neural correlates of maladaptive pain facilitation - such as the cortico-amygdalar connectivity observed in this study - could be assessed prior to surgery and identify individuals at risk of developing chronic post-operative pain.

Emotional modulation of pain allows the organism to adjust behaviour in a contextappropriate manner and maximizes adaptive learning processes. However, individuals differ widely in the degree to which this modulation occurs. As with other adaptive affective responses like fear and anxiety, some individuals' level of emotional facilitation of pain can be maladaptive, leading to long term suffering. As such, it is critical to understand the biological mechanisms that give rise to these responses so that we can understand the processes that confer risk or resilience for more intrusive and long-lasting pain states. Here we demonstrate that functional connectivity of the amygdala with cortical regions involved in sensory and motor responses is associated with pain facilitation by negative emotional states. Future work is needed to determine the degree to which these patterns of functional connectivity represent biological markers for longer term patterns of maladaptive pain coping, including the development of chronic pain in the wake of physical trauma.

\section{Acknowledgments}

The authors declare no conflicts of interest.

WG is funded by a Leverhulme Early Career Fellowship, NRR by a Magdalen Vernon Studentship from the Department for Psychology and Clinical Language Sciences, University of Reading, and RH by a joint NHS-UoR CASE studentship. TVS was funded by a Marie Curie 
International Incoming Fellowship from the European Commission and a British Academy/Leverhulme Small Project Grant. 


\section{References}

[1] Akirav I, Richter-Levin GJD-R. Factors that determine the non-linear amygdala influence on hippocampus-dependent memory. 2006;4(1):dose-response. 004.001. 003. Akirav.

[2] Althaus A, Arranz Becker O, Neugebauer E. Distinguishing between pain intensity and pain resolution: using acute post-surgical pain trajectories to predict chronic postsurgical pain. European journal of pain (London, England) 2014;18(4):513-521.

[3] Arnold BS, Alpers GW, Suss H, Friedel E, Kosmutzky G, Geier A, Pauli P. Affective pain modulation in fibromyalgia, somatoform pain disorder, back pain, and healthy controls. European journal of pain (London, England) 2008;12(3):329-338.

[4] Bair MJ, Robinson RL, Katon W, Kroenke K. Depression and pain comorbidity: a literature review. Arch Intern Med 2003;163(20):2433-2445.

[5] Beck AT, Steer RA, Brown GK. Manual for the Beck Depression Inventory-II. San Antonio, TX: Psychological Corporation, 1996.

[6] Becker S, Gandhi W, Elfassy NM, Schweinhardt P. The role of dopamine in the perceptual modulation of nociceptive stimuli by monetary wins or losses. Eur J Neurosci 2013.

[7] Brandsborg B, Nikolajsen L, Kehlet H, Jensen TS. Chronic pain after hysterectomy. Acta anaesthesiologica Scandinavica 2008;52(3):327-331.

[8] Buneo CA, Jarvis MR, Batista AP, Andersen RA. Direct visuomotor transformations for reaching. Nature 2002;416(6881):632-636.

[9] Bushnell MC, Ceko M, Low LA. Cognitive and emotional control of pain and its disruption in chronic pain. Nature reviews Neuroscience 2013;14(7):502-511.

[10] Camacho EM, Verstappen SM, Chipping J, Symmons DP. Learned helplessness predicts functional disability, pain and fatigue in patients with recent-onset inflammatory polyarthritis. Rheumatology 2013;52(7):1233-1238. 
[11] Carroll L, Cassidy JD, Cote P. Depression as a risk factor for onset of an episode of troublesome neck and low back pain. Pain 2004;107(1-2):134-139.

[12] Chudler EH, Anton F, Dubner R, Kenshalo DR, Jr. Responses of nociceptive SI neurons in monkeys and pain sensation in humans elicited by noxious thermal stimulation: effect of interstimulus interval. Journal of neurophysiology 1990;63(3):559-569.

[13] Coen SJ, Aziz Q, Yaguez L, Brammer M, Williams SC, Gregory LJ. Effects of attention on visceral stimulus intensity encoding in the male human brain. Gastroenterology 2008;135(6):2065-2074, 2074.e2061.

[14] Corder G, Ahanonu B, Grewe BF, Wang D, Schnitzer MJ, Scherrer G. An amygdalar neural ensemble that encodes the unpleasantness of pain. Science (New York, NY) 2019;363(6424):276-281.

[15] Corradi-Dell'Acqua C, Fink GR, Weidner R. Selecting category specific visual information: Top-down and bottom-up control of object based attention. Consciousness and cognition 2015;35:330-341.

[16] Davidson RJ. Anxiety and affective style: role of prefrontal cortex and amygdala. Biol Psychiatry 2002;51(1):68-80.

[17] Davis M. The role of the amygdala in fear and anxiety. Annual review of neuroscience $1992 ; 15(1): 353-375$.

[18] Davis M. Anatomic and physiologic substrates of emotion in an animal model. J Clin Neurophysiol 1998;15(5):378-387.

[19] Fogassi L, Luppino G. Motor functions of the parietal lobe. Current opinion in neurobiology 2005;15(6):626-631. 
[20] Gao YJ, Ren WH, Zhang YQ, Zhao ZQ. Contributions of the anterior cingulate cortex and amygdala to pain- and fear-conditioned place avoidance in rats. Pain 2004;110(12):343-353.

[21] Gerbershagen HJ, Dagtekin O, Rothe T, Heidenreich A, Gerbershagen K, Sabatowski R, Petzke F, Ozgur E. Risk factors for acute and chronic postoperative pain in patients with benign and malignant renal disease after nephrectomy. European journal of pain (London, England) 2009;13(8):853-860.

[22] Greenspan JD, Lee RR, Lenz FA. Pain sensitivity alterations as a function of lesion location in the parasylvian cortex. Pain 1999;81(3):273-282.

[23] Harrison R, Zeidan F, Kitsaras G, Ozcelik D, Salomons TV. Trait Mindfulness Is Associated With Lower Pain Reactivity and Connectivity of the Default Mode Network. The journal of pain : official journal of the American Pain Society 2019;20(6):645-654.

[24] Hartmann KE, Ma C, Lamvu GM, Langenberg PW, Steege JF, Kjerulff KH. Quality of life and sexual function after hysterectomy in women with preoperative pain and depression. Obstetrics and gynecology 2004;104(4):701-709.

[25] Hashmi JA, Baliki MN, Huang L, Baria AT, Torbey S, Hermann KM, Schnitzer TJ, Apkarian AV. Shape shifting pain: chronification of back pain shifts brain representation from nociceptive to emotional circuits. Brain : a journal of neurology 2013;136(9):2751-2768.

[26] Jackson T, Tian P, Wang Y, lezzi T, Xie W. Toward Identifying Moderators of Associations Between Presurgery Emotional Distress and Postoperative Pain Outcomes: A MetaAnalysis of Longitudinal Studies. The journal of pain : official journal of the American Pain Society 2016;17(8):874-888. 
[27] Jenkinson $M$, Bannister $P$, Brady $M$, Smith S. Improved optimization for the robust and accurate linear registration and motion correction of brain images. Neurolmage $2002 ; 17(2): 825-841$.

[28] Jenkinson M, Beckmann CF, Behrens TE, Woolrich MW, Smith SM. Fsl. Neurolmage $2012 ; 62(2): 782-790$

[29] Kalaska JF, Crammond DJ. Cerebral cortical mechanisms of reaching movements. Science (New York, NY) 1992;255(5051):1517-1523.

[30] Kamping S, Andoh J, Bomba IC, Diers M, Diesch E, Flor H. Contextual modulation of pain in masochists: involvement of the parietal operculum and insula. Pain 2016;157(2):445.

[31] Kamping S, Bomba IC, Kanske P, Diesch E, Flor H. Deficient modulation of pain by a positive emotional context in fibromyalgia patients. Pain 2013;154(9):1846-1855.

[32] Keefe FJ, Rumble ME, Scipio CD, Giordano LA, Perri LM. Psychological aspects of persistent pain: current state of the science. The Journal of Pain 2004;5(4):195-211.

[33] Kenntner-Mabiala R, Pauli P. Affective modulation of brain potentials to painful and nonpainful stimuli. Psychophysiology 2005;42(5):559-567.

[34] Khatibi A, Vachon-Presseau E, Schrooten M, Vlaeyen J, Rainville P. Attention effects on vicarious modulation of nociception and pain. Pain 2014;155(10):2033-2039.

[35] Lang PJ. International affective picture system (IAPS): Affective ratings of pictures and instruction manual. Technical report 2005.

[36] Lang PJG, FL: The Center for Research in Psychophysiology, University of Florida. Selfassessment manikin. 1980.

[37] Lang PJJTR. International affective picture system (IAPS): Digitized photographs, instruction manual and affective ratings. 2005. 
[38] Lapate RC, Lee H, Salomons TV, van Reekum CM, Greischar LL, Davidson RJ. Amygdalar function reflects common individual differences in emotion and pain regulation success. Journal of Cognitive Neuroscience 2012;24(1):148-158.

[39] Lavand'homme P. Transition from acute to chronic pain after surgery. Pain 2017;158 Suppl 1:S50-s54.

[40] LeDoux J. Neurobiology of emotion. Mind and brain: Dialogues in cognitive neuroscience: Cambridge University Press, 1986.

[41] LeDoux J. The amygdala. Current biology : CB 2007;17(20):R868-874.

[42] LeDoux JE. Emotion: Clues from the brain. Annual review of psychology 1995;46(1):209235.

[43] LeDoux JE. Emotion, memory and the brain. Scientific American 1997;7(1):68-75.

[44] Lépine JP, Briley M. The epidemiology of pain in depression. Human Psychopharmacology: Clinical and Experimental 2004;19(S1).

[45] Masselin-Dubois A, Attal N, Fletcher D, Jayr C, Albi A, Fermanian J, Bouhassira D, Baudic S. Are psychological predictors of chronic postsurgical pain dependent on the surgical model? A comparison of total knee arthroplasty and breast surgery for cancer. The journal of pain : official journal of the American Pain Society 2013;14(8):854-864.

[46] Meagher MW, Arnau RC, Rhudy JL. Pain and emotion: effects of affective picture modulation. Psychosom Med 2001;63(1):79-90.

[47] Morrison I, Perini I, Dunham J. Facets and mechanisms of adaptive pain behavior: predictive regulation and action. Front Hum Neurosci 2013;7:755.

[48] Neugebauer V, Li W, Bird GC, Han JS. The amygdala and persistent pain. Neuroscientist $2004 ; 10(3): 221-234$ 
[49] Nicholson AA, Friston KJ, Zeidman P, Harricharan S, McKinnon MC, Densmore M, Neufeld RWJ, Theberge J, Corrigan F, Jetly R, Spiegel D, Lanius RA. Dynamic causal modeling in PTSD and its dissociative subtype: Bottom-up versus top-down processing within fear and emotion regulation circuitry. Human brain mapping 2017;38(11):55515561.

[50] Phelps EA, LeDoux JE. Contributions of the amygdala to emotion processing: from animal models to human behavior. Neuron 2005;48(2):175-187.

[51] Pinheiro MB, Ferreira ML, Refshauge K, Colodro-Conde L, Carrillo E, Hopper JL, Ordonana JR, Ferreira PH. Genetics and the environment affect the relationship between depression and low back pain: a co-twin control study of Spanish twins. Pain 2015;156(3):496-503.

[52] Ploner M, Freund HJ, Schnitzler A. Pain affect without pain sensation in a patient with a postcentral lesion. Pain 1999;81(1-2):211-214.

[53] Price DD. Psychological and neural mechanisms of the affective dimension of pain. Science (New York, NY) 2000;288(5472):1769-1772.

[54] Rainville P. Brain mechanisms of pain affect and pain modulation. Current opinion in neurobiology 2002;12(2):195-204.

[55] Rainville P, Duncan GH, Price DD, Carrier B, Bushnell MC. Pain affect encoded in human anterior cingulate but not somatosensory cortex. Science (New York, NY) 1997;277(5328):968-971.

[56] Rao NG, Donoghue JP. Cue to action processing in motor cortex populations. Journal of neurophysiology 2014;111(2):441-453.

[57] Rhudy JL, Meagher MW. Fear and anxiety: divergent effects on human pain thresholds. Pain 2000;84(1):65-75. 
[58] Rhudy JL, Meagher MW. The role of emotion in pain modulation. Current Opinion in Psychiatry 2001;14(3):241-245.

[59] Richez B, Ouchchane L, Guttmann A, Mirault F, Bonnin M, Noudem Y, Cognet V, Dalmas AF, Brisebrat L, Andant N, Soule-Sonneville S, Dubray C, Duale C, Schoeffler P. The Role of Psychological Factors in Persistent Pain After Cesarean Delivery. The journal of pain : official journal of the American Pain Society 2015;16(11):1136-1146.

[60] Rizzolatti G, Fogassi L, Gallese V. Parietal cortex: from sight to action. Current opinion in neurobiology 1997;7(4):562-567.

[61] Roy AK, Shehzad Z, Margulies DS, Kelly AM, Uddin LQ, Gotimer K, Biswal BB, Castellanos FX, Milham MP. Functional connectivity of the human amygdala using resting state fMRI. Neurolmage 2009;45(2):614-626.

[62] Roy M, Lebuis A, Hugueville L, Peretz I, Rainville P. Spinal modulation of nociception by music. European Journal of Pain 2012;16(6):870-877.

[63] Roy M, Lebuis A, Peretz I, Rainville P. The modulation of pain by attention and emotion: a dissociation of perceptual and spinal nociceptive processes. European journal of pain (London, England) 2011;15(6):641.e641-610.

[64] Roy M, Piché M, Chen J-I, Peretz I, Rainville P. Cerebral and spinal modulation of pain by emotions. Proceedings of the National Academy of Sciences 2009;106(49):2090020905.

[65] Sadler KE, McQuaid NA, Cox AC, Behun MN, Trouten AM, Kolber BJ. Divergent functions of the left and right central amygdala in visceral nociception. 2017;158(4):747-759.

[66] Schwartz N, Temkin P, Jurado S, Lim BK, Heifets BD, Polepalli JS, Malenka RC. Chronic pain. Decreased motivation during chronic pain requires long-term depression in the nucleus accumbens. Science (New York, NY) 2014;345(6196):535-542. 
[67] Scott SH. The role of primary motor cortex in goal-directed movements: insights from neurophysiological studies on non-human primates. Current opinion in neurobiology $2003 ; 13(6): 671-677$

[68] Smith SM. Fast robust automated brain extraction. Human brain mapping $2002 ; 17(3): 143-155$

[69] Spielberger C, Gorsuch R, Lushene R, Vagg P, Jacobs G. Manual for the State-Trait Anxiety Inventory (form Y). Palo Alto (CA): Consulting Psychologists Press, 1983.

[70] Steiger JH. Tests for comparing elements of a correlation matrix. Psychological bulletin $1980 ; 87(2): 245$.

[71] Strigo IA, Simmons AN, Matthews SC, Craig AD, Paulus MP. Association of major depressive disorder with altered functional brain response during anticipation and processing of heat pain. Arch Gen Psychiatry 2008;65(11):1275-1284.

[72] Sullivan MJL, Bishop SR, Pivik J. The Pain Catastrophizing Scale: Development and validation. Psychological Assessment 1995;7(4):524-532.

[73] Theunissen M, Peters ML, Bruce J, Gramke HF, Marcus MA. Preoperative anxiety and catastrophizing: a systematic review and meta-analysis of the association with chronic postsurgical pain. The Clinical journal of pain 2012;28(9):819-841.

[74] Thompson JM, Neugebauer V. Cortico-limbic pain mechanisms. Neuroscience letters 2018

[75] Tolle TR, Kaufmann T, Siessmeier T, Lautenbacher S, Berthele A, Munz F, Zieglgansberger W, Willoch F, Schwaiger M, Conrad B, Bartenstein P. Region-specific encoding of sensory and affective components of pain in the human brain: a positron emission tomography correlation analysis. Annals of neurology 1999;45(1):40-47. 
[76] Treede RD, Apkarian AV, Bromm B, Greenspan JD, Lenz FA. Cortical representation of pain: functional characterization of nociceptive areas near the lateral sulcus. Pain 2000;87(2):113-119.

[77] Vandyk AD, Brenner I, Tranmer J, Van Den Kerkhof E. Depressive symptoms before and after elective hysterectomy. Journal of obstetric, gynecologic, and neonatal nursing : JOGNN 2011;40(5):566-576.

[78] Villemure C, Bushnell MC. Cognitive modulation of pain: how do attention and emotion influence pain processing? Pain 2002;95(3):195-199.

[79] Villemure C, Bushnell MC. Mood Influences Supraspinal Pain Processing Separately from Attention. Journal of Neuroscience 2009;29(3):705-715.

[80] Villemure C, Slotnick BM, Bushnell MC. Effects of odors on pain perception: deciphering the roles of emotion and attention. Pain 2003;106(1-2):101-108.

[81] Weinrib AZ, Azam MA, Birnie KA, Burns LC, Clarke H, Katz J. The psychology of chronic post-surgical pain: new frontiers in risk factor identification, prevention and management. British journal of pain 2017;11(4):169-177.

[82] Wiech K. Deconstructing the sensation of pain: The influence of cognitive processes on pain perception. Science (New York, NY) 2016;354(6312):584-587.

[83] Wiech K, Ploner M, Tracey I. Neurocognitive aspects of pain perception. Trends Cogn Sci 2008;12(8):306-313.

[84] Wiech K, Tracey I. The influence of negative emotions on pain: behavioral effects and neural mechanisms. Neurolmage 2009;47(3):987-994.

[85] Williams EJ. The comparison of regression variables. Journal of the Royal Statistical Society Series B (Methodological) 1959:396-399. 
[86] Williamson A, Hoggart B. Pain: a review of three commonly used pain rating scales. Journal of clinical nursing 2005;14(7):798-804.

[87] Yerkes RM, Dodson JD. The relation of strength of stimulus to rapidity of habitformation. Journal of comparative neurology and psychology 1908;18(5):459-482.

[88] Zhang Y, Brady M, Smith S. Segmentation of brain MR images through a hidden Markov random field model and the expectation-maximization algorithm. IEEE transactions on medical imaging 2001;20(1):45-57.

[89] Zubieta JK, Smith YR, Bueller JA, Xu Y, Kilbourn MR, Jewett DM, Meyer CR, Koeppe RA, Stohler CS. Regional mu opioid receptor regulation of sensory and affective dimensions of pain. Science (New York, NY) 2001;293(5528):311-315. 


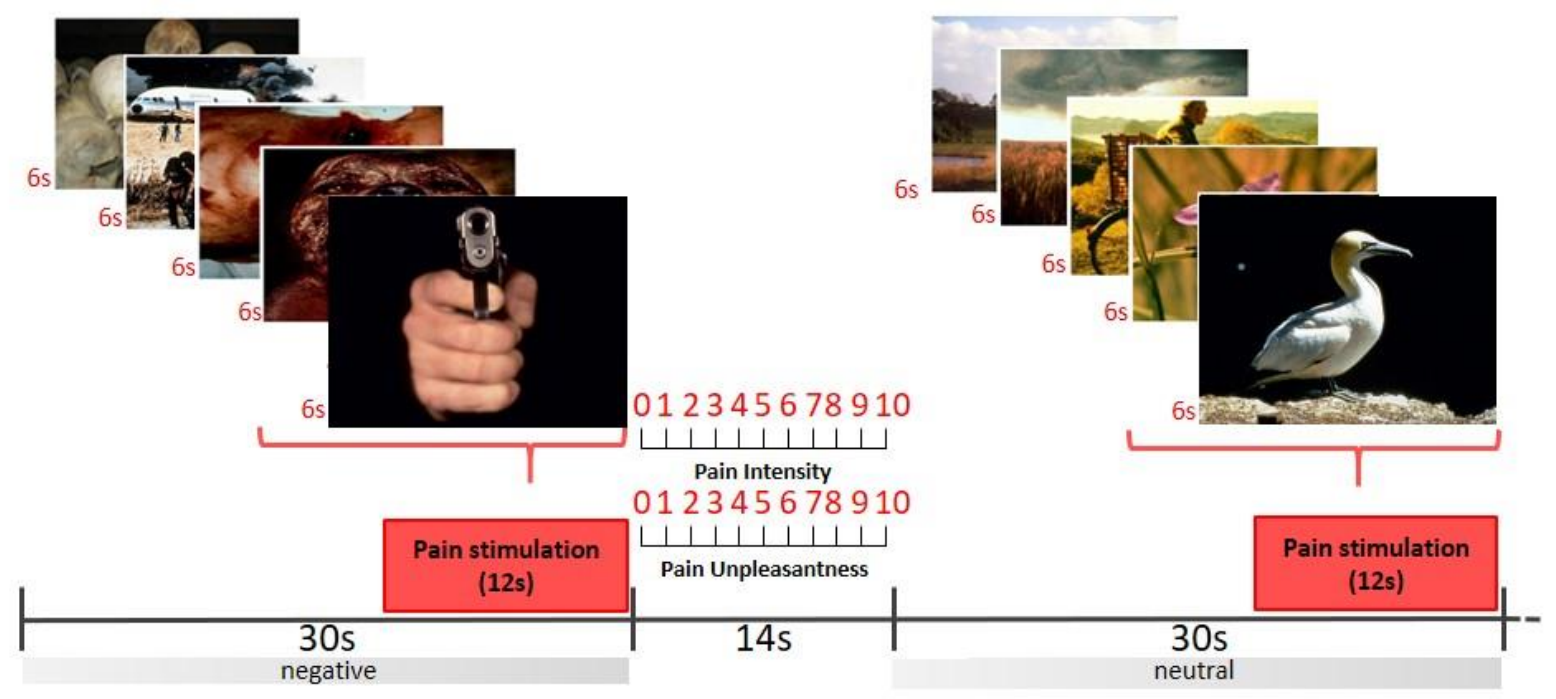

Figure 1. Example trial of the emotional pain-modulation task. In each trial five pictures of either negative or neutral valence were presented for 6 seconds each. Simultaneous to the presentation of the final two images, the participants received a 12 second thermal pain stimulus applied to their calf. Subsequently, participants were asked to rate the pain intensity they had felt during the thermal stimulation (within 7 seconds), and finally the pain unpleasantness they had perceived (within 7 seconds). Negative and neutral stimuli were presented in alternating fashion. 


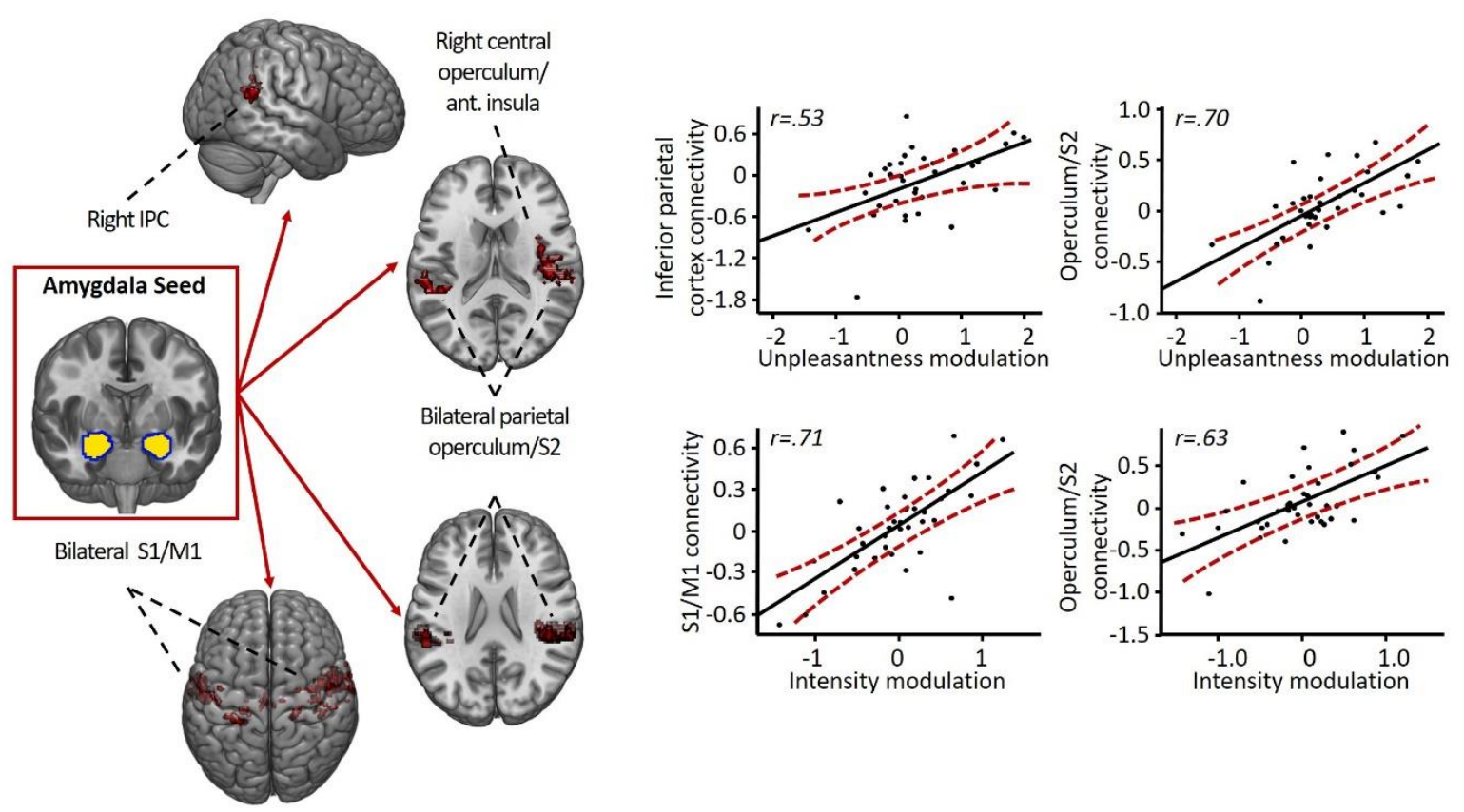

Figure 2. Connectivity of the amygdala to the inferior parietal cortex (IPC), S1/M1, and S2/operculum at rest is associated with the individual amplitude to modulate pain unpleasantness and intensity by emotion. 

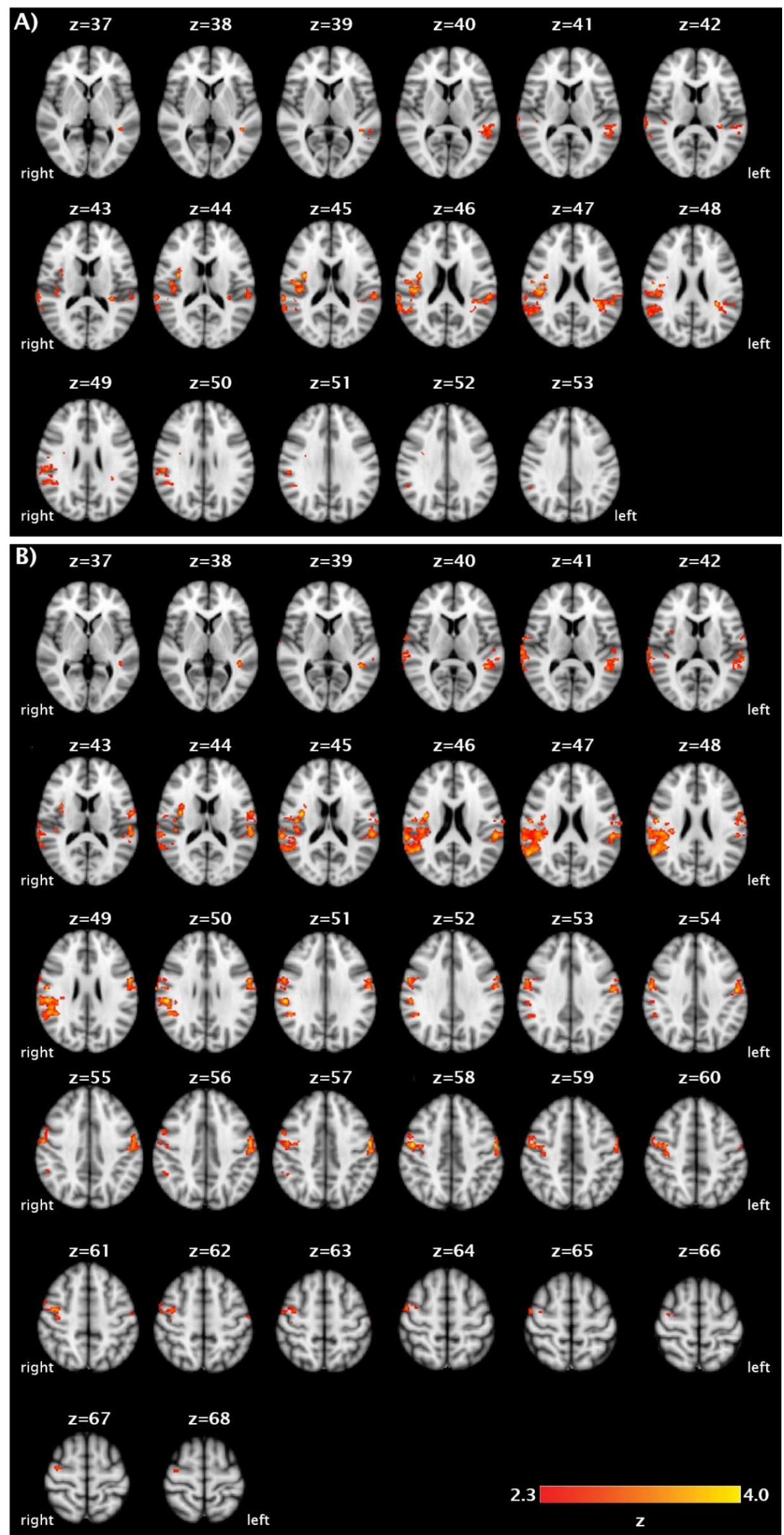
Figure S1. Statistical maps of amygdalar connectivity correlated with emotional pain modulation of pain unpleasantness (A) and pain intensity (B). Voxel-based threshold $z>2.3$, cluster corrected for spatial extent $(p<0.05)$ across the whole brain. Images are displayed in radiological convention, i.e., right side of the brain is on the left. Z-Coordinates for all axial slides are given in MNI space.

a)

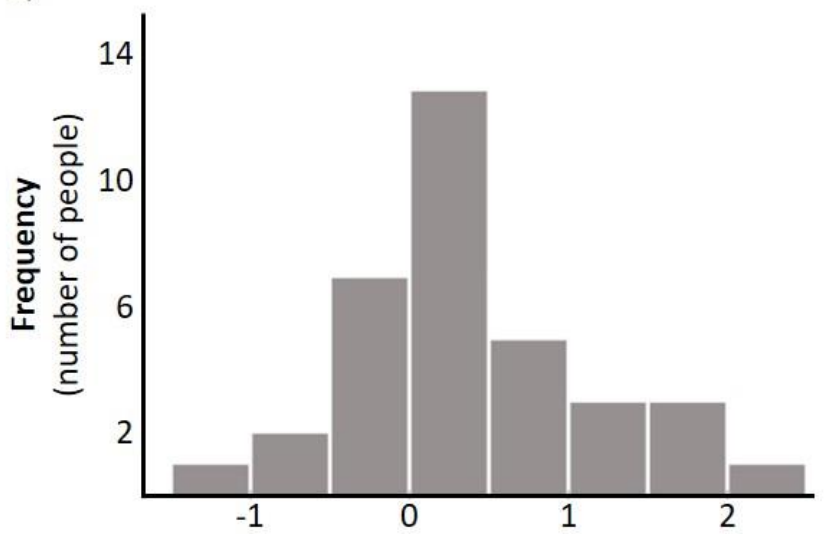

Pain Unpleasantness Modulation (NRS, negative-neutral) b)

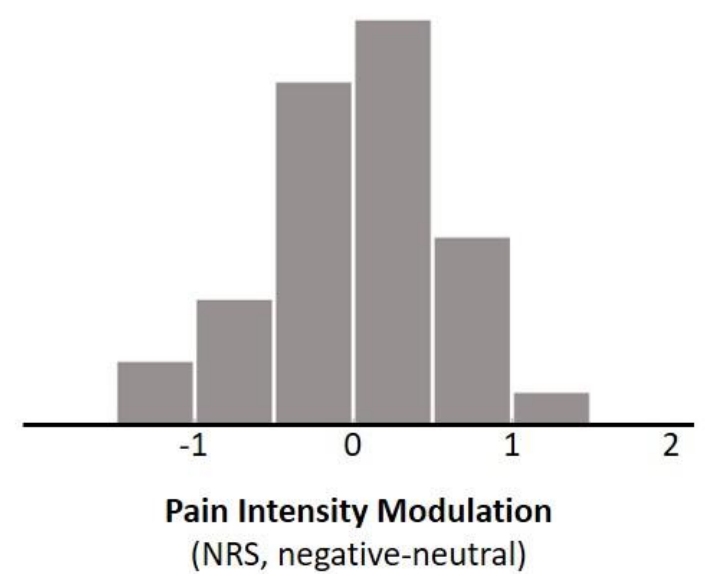

Figure S2. Histograms showing the range of individual magnitudes of emotional pain modulation for a) pain unpleasantness and $b$ ) pain intensity ratings by pictures of negative valence. The $\mathrm{x}$-axis displays the difference in pain ratings for pictures of negative and neutral valence - positive values represent higher pain ratings in the negative versus the neutral condition; conversely, negative values represent lower pain ratings in the negative versus the neutral condition. The $y$-axis displays the number of participants showing the respective magnitude of emotional pain modulation.

Table 1 Brain regions associated with emotional modulation of pain intensity or pain unpleasantness

\begin{tabular}{|c|c|c|c|c|c|c|}
\hline \multirow[t]{2}{*}{ Seed } & \multirow{2}{*}{$\begin{array}{l}\text { Behavioural } \\
\text { Measure }\end{array}$} & \multirow[t]{2}{*}{ Brain region } & \multicolumn{3}{|c|}{$\underline{\text { MNI coordinates }}$} & \multirow{2}{*}{$\begin{array}{l}\text { Peak } \\
\text { z-score }\end{array}$} \\
\hline & & & $\mathrm{x}$ & Y & Z & \\
\hline & & Positive correlation & & & & \\
\hline \multirow[t]{3}{*}{ Bil. Amygdala } & Pain UP & Parietal/central operculum (R) & 36 & -4 & 18 & 4.00 \\
\hline & & Parietal operculum (L) & -32 & -40 & 24 & 3.74 \\
\hline & & Inferior parietal cortex $(\mathrm{R})$ & 64 & -34 & 18 & 3.25 \\
\hline \multirow[t]{4}{*}{ Bil. Amygdala } & Pain Intensity & Parietal operculum (R) & 54 & -28 & 28 & 4.09 \\
\hline & & Parietal operculum (L) & -56 & -32 & 20 & 3.81 \\
\hline & & $\mathrm{M} 1 / \mathrm{S} 1(\mathrm{R})$ & 48 & -8 & 44 & 4.08 \\
\hline & & $\mathrm{M} 1 / \mathrm{S} 1$ (L) & -54 & -8 & 42 & 3.80 \\
\hline
\end{tabular}


Table S1 Correlations between parameter estimates and psychological questionnaires.

\begin{tabular}{lcccccc}
\hline & \multicolumn{2}{c}{ PCS } & \multicolumn{2}{c}{ BDI-II } & \multicolumn{2}{c}{ STAI } \\
& $\mathrm{r}$ & $\mathrm{p}$ & $\mathrm{r}$ & $\mathrm{p}$ & $\mathrm{r}$ & $\mathrm{p}$ \\
\hline Operculum (UP cluster) & -0.15 & 0.39 & -0.10 & 0.56 & -0.13 & 0.47 \\
Operculum (INT cluster) & -0.27 & 0.11 & -0.20 & 0.24 & -0.31 & 0.06 \\
Inferior parietal cortex & -0.13 & 0.46 & -0.23 & 0.19 & -0.10 & 0.56 \\
M1/S1 & -0.27 & 0.11 & -0.19 & 0.25 & -0.18 & 0.30 \\
\hline
\end{tabular}

PCS Pain Catastrophizing Scale; BDI Beck's Depression Inventory; STAI State-Trait Anxiety Inventory; UP cluster parameter estimates were used of the amygdala connectivity to the operculum cluster associated with pain unpleasantness modulation; INT cluster parameter estimates were used of the amygdala connectivity to the operculum cluster associated with pain intensity modulation; M1 primary motor cortex; $\mathbf{S 1}$ primary somatosensory cortex; $\mathbf{r}$ Pearson's correlation coefficient; $\mathbf{p}$ pvalue. 